\title{
Cuirass respirator treatment of chronic respiratory failure in scoliotic patients
}

\author{
P. W. J. WIERS, R. LE COUlTRE, O. T. DALlingA, W. VAN DIJL, A. F. MEINESZ, \\ AND H. J. SLUITER
}

From the Respiratory Care Unit, University Hospital, Groningen, The Netherlands

Wiers, P. W. J., le Coultre, R., Dallinga, O. T., van Dijl, W., Meinesz, A. F., and Sluiter, H. J. (1977). Thorax, 32, 221-228. Cuirass respirator treatment of chronic respiratory failure in scoliotic patients. The results are reported of domiciliary cuirass respirator treatment, using tailor-made shells, in four patients with severe thoracic scoliosis. Three of the patients had suffered from poliomyelitis. All complained of increasing dyspnoea on exertion, ultimately interfering with almost every activity of daily life; three patients had severe acute respiratory failure necessitating urgent admission to the Respiratory Care Unit. Right heart failure was present in two. Two patients required mechanical treatment via an endotracheal tube. All the patients were discharged home with a cuirass respirator. Standard type shells were used initially with low efficiency due to the poor fit of the cuirass shell to the deformed thoracic cage. Tailor-made shells were constructed from polyester reinforced with glass fibre, modelled on plaster casts of the thoracic cage. Subjectively the patients improved greatly and were able to resume and increase many activities. One patient committed suicide for reasons unconnected with treatment but the other three patients have been doing well from the time the cuirass respirator treatment was started, respectively, 3, 6, and 10 years ago. This treatment seems particularly effective in younger patients with severe paralytic scoliosis and cardiorespiratory failure, although the possibility of using it in older patients suffering from scoliosis of other aetiology should certainly be explored.

Scoliosis of the thoracic spine causes a decrease in lung volumes and regional disturbances in ventilation-perfusion relationships which may lead to hypoxaemia and, in severe cases, to hypercapnia, respiratory acidosis, pulmonary hypertension, and cor pulmonale (Bergofsky et al., 1959; Caro and Dubois, 1961; Dollery et al., 1965; Towers and Zorab, 1969; Littler et al., 1973). The work of breathing is increased because of the low compliance of the thoracic wall, and the respiratory muscles perform at a low level of efficiency (Bergofsky et al., 1959; Prime and Zorab, 1969). Complicating factors may be impaired function of the respiratory muscles, for example, diaphragmatic paralysis in poliomyelitis (Scadding and Zorab, 1969; Lane et al., 1974; Newson Davis et al., 1976) and bacterial bronchial infections, the latter often in patients with co-existent airways obstruction (Dollery et al., 1965). Endotracheal intubation and short-term mechanical ventilation are often successful in the treatment of an acute episode. Mortality is high in the first few years after an episode of cardiorespiratory failure, even with optimal conservative treatment (Towers and Zorab, 1969). To improve the long-term prognosis several possibilities have been explored such as intermittent positive pressure breathing (IPPB) (Bergofsky et al., 1959; Lane et al., 1974), tracheostomy (Bruderman and Stein, 1961), and treatment with a cuirass respirator (Newson Davis et $a l .$, 1976). The standard type of cuirass shell fits poorly in scoliotic patients and therefore often fails to improve the condition. We have used tailor-made cuirass shells in severely scoliotic patients. This paper describes the results obtained in four patients who were treated in this way.

\section{Patients and methods}

In the last 20 years over 2000 patients with severe respiratory failure have been treated in the Respiratory Care Unit (RCU) of the University 
Hospital of Groningen. In 17 of these, severe thoracic scoliosis (grade IV, Scadding and Zorab (1969)) was the main cause of the respiratory failure. Thirteen of the 17 patients were treated with mechanical ventilation during the acute episode; five died during this episode and eight patients survived. Chronic respirator treatment was judged not to be indicated in five patients; three of them died within three years of the acute episode. Three patients were discharged with a permanent tracheostomy. One died eight years later. The cause of death in all patients was severe (cardio-) respiratory failure. The present paper discusses four patients who were treated with a cuirass respirator after recovery from the acute episode; two had respirator treatment during the acute episode, one patient had a tracheostomy, and one patient was treated conservatively at the RCU. The Table gives some details of these patients.

Examination on admission included a detailed history and physical examination, chest radiograph, electrocardiogram, arterial blood analysis (Po $_{2}$ and $\mathrm{PCO}_{2}$ membrane electrodes; $\mathrm{pH}$ : glass electrode, Radiometer, Copenhagen), bacteriological examination of sputum (Mulder, 1956), and detailed biochemical studies. Cor pulmonale was diagnosed in the presence of symptoms and signs of right heart failure and changes in the electrocardiogram or vectorcardiogram (Friedman, 1971). A diagnosis of airways obstruction was made on the basis of history, physical examination, and lung function data (Orie, 1961; van der Lende, 1969; van der Lende, 1977).

Lung function data were obtained either before or after recovery; a Lode spirometer type D $53 \mathrm{R}$ was used. Predicted values were calculated according to Tammeling (1961), using arm span instead of height (Hepper et al., 1965). Respirator treatment in the acute phase was carried out via an endotracheal tube (Portex ${ }^{\circledR}$ ) during the first week; if respirator treatment was still necessary after one week a tracheostomy was performed. We used Lundia, Bennett PR-2, Engström 150 or 200, and Cape mark II respirators.

The cuirass respirators used after recovery from the acute episode were either Dräger or Monaghan 170C.

Cuirass shells are available in different sizes. To perform well the shell must fit closely to the thoracic wall, otherwise the pressure changes are insufficient to ventilate the lungs. Leakage of air and pressure sores must be prevented. Standard cuirass shells seldom meet these requirements in severe scoliotic patients (Fountain et al., 1973). With the cooperation of several departments of the University Hospital, tailor-made cuirass shells $\overrightarrow{\overline{\vec{N}}}$ were made using polyester reinforced with glasso fibre, and a foam rubber rim; they were modelled등 from a plaster cast made of the patient's thoracic $\overline{\bar{c}}$ cage. Figures 1 and 2 show the two shells that were used.

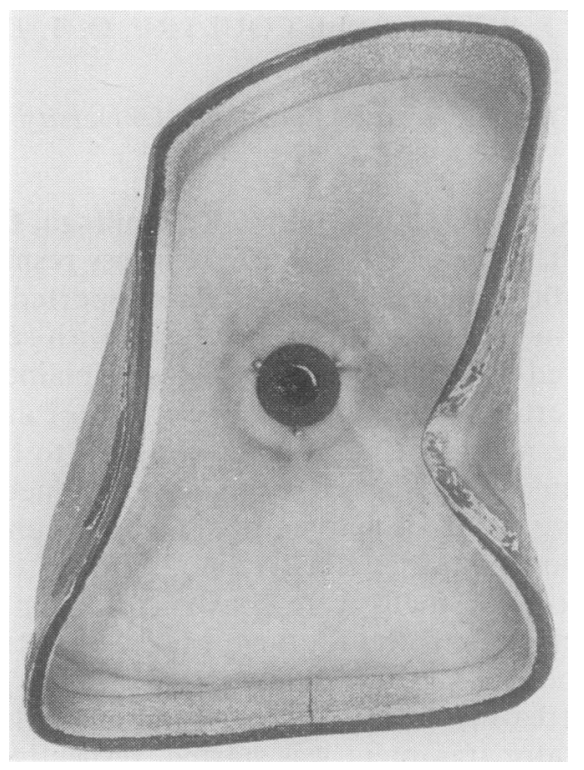

Fig. 1 Tailor-made cuirass shell, inside view, showing the marked asymmetry of the thoracic cage.

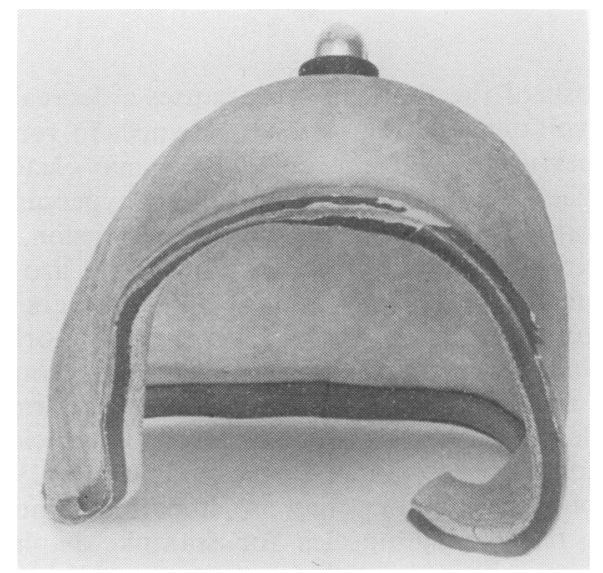

Fig. 2 Tailor-made cuirass shell, outside view. The rim shows changes due to wear and tear.

\section{Results}

Three of the four patients are alive, respectively, 10,6 , and 3 years after the cuirass respiratoro 


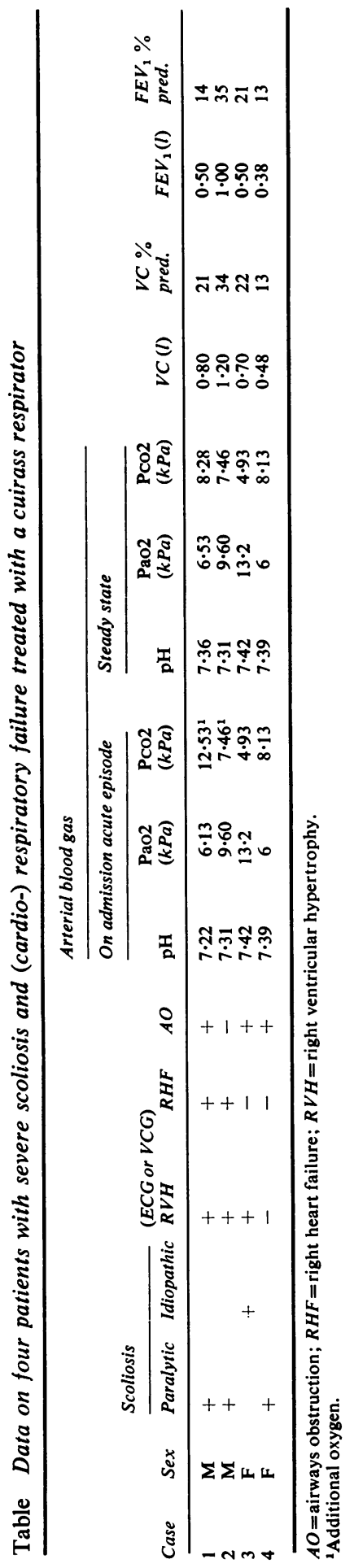


treatment was started; one patient committed suicide. All four patients felt much better after cuirass treatment and were able to carry out more duties than previously. Co-existent airways obstruction was present in three of the four patients. Lung function results were in accordance with the severity of the scoliosis (Weber et al., 1975); mean vital capacity (VC) was $23 \%$ and mean forced expiratory volume in one second $\left(\mathrm{FEV}_{1}\right) 21 \%$ of the predicted value. In general, the patients accepted a lower breathing frequency of the cuirass respirator than their usual spontaneous respiratory rate; the tidal volume tended to rise (Fig. 3).

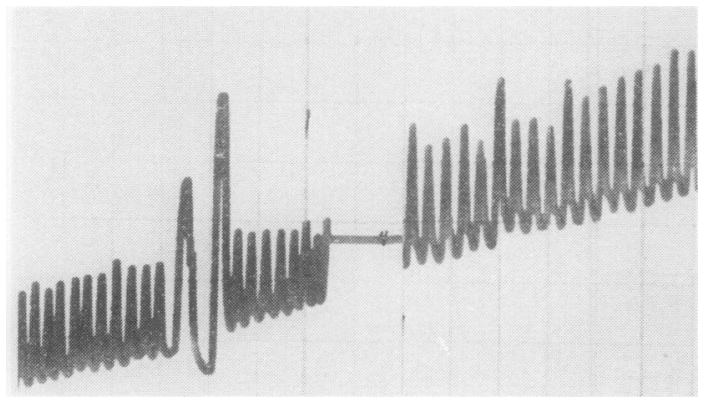

Fig. 3 Case 2 Spirographic tracing. Left: spontaneous breathing and a vital capacity manoeuvre; right: during cuirass respirator treatment.

\section{Case histories}

The history of case 2 will be described first, because it illustrates best the evolution of the treatment towards the tailor-made cuirass shell.

\section{CASE 2}

This patient had poliomyelitis in 1956 when he was 4 years of age. $\mathrm{He}$ was treated at that time in a tank respirator for six weeks, discharged home two months later, and was thereafter lost to follow-up. In 1970, aged 18, he visited the outpatient pulmonary diseases department complaining of severe dyspnoea on exertion, headache, fatigue, and insomnia. He was doing well in high school and had until that time managed to ride a bicycle. He lived with his family in a first-floor apartment. He was a slender, intelligent young man with severe thoracic scoliosis (Fig. 4), widespread pareses, and atrophy of the muscles of the trunk, limbs, and intercostal muscles. No treatment was advised. A few months later his exercise tolerance had diminished still further and we decided to try cuirass respirator treatment during the night, using a conventional shell, to improve

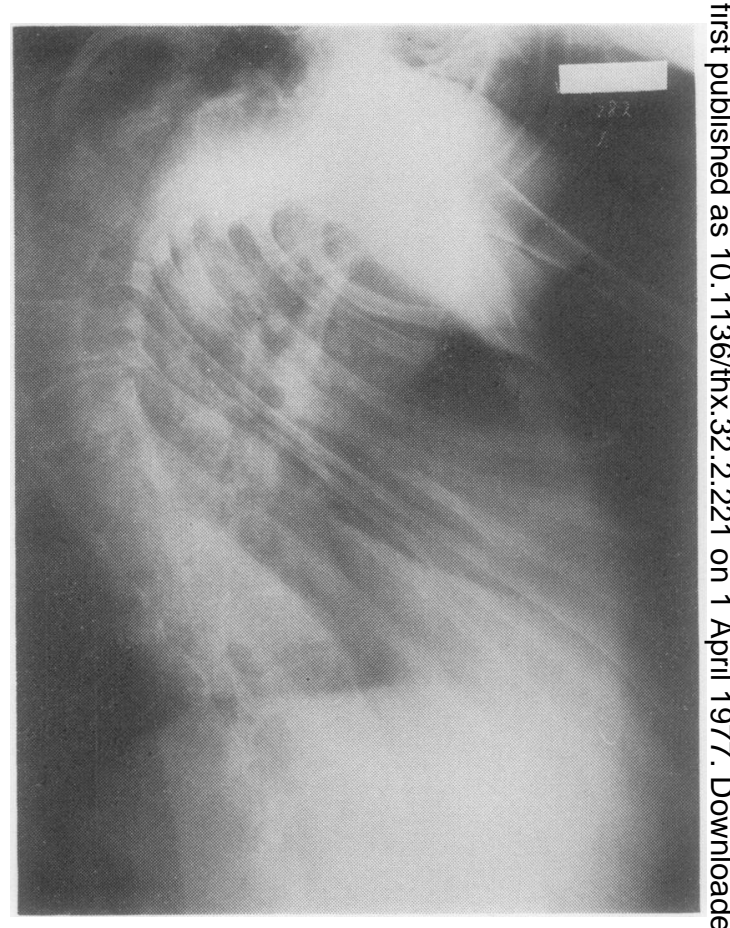

Fig. 4 Case 2 Chest radiograph showing marked deformity of the thorax.

his exercise tolerance. A slight improvement waș noted and the patient was able to resume some activities. One year later he was admitted to theo RCU with cardiorespiratory failure $\left(\mathrm{Po}_{2}: 6 \mathrm{kPa} \stackrel{\mathrm{m}}{\sim}\right.$

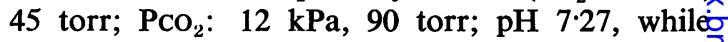
breathing oxygen, 0.5 litre per minute, via a nasa catheter). No bacterial bronchial infection coulB be demonstrated. Mechanical ventilation was started via an endotracheal tube and the patient's condition improved rapidly. The endotracheal tube was removed after a few days and mechanica $\underline{P}$ ventilation was continued by means of a tank respirator, first day and night and, in a later phase, only during the night. In the meantime a kind of corset-cuirass was constructed (Figs 5 and 6) that encased the patient's body with the exception of the head and limbs. Although it worked efficiently it was ungainly and the patient had to be helpeç when putting on this apparatus, which he con $\stackrel{?}{4}$ sidered to be a major disadvantage.

He went home, using the apparatus, finishe $\bar{\phi}$ college, and started to read psychology. His exer cise tolerance was greatly improved. Four year $\overrightarrow{8}$ later, in 1974, when his cuirass shell showed signs of wear and tear, a new tailor-made shell was constructed (Fig. 7) modelled on a plaster cast of 


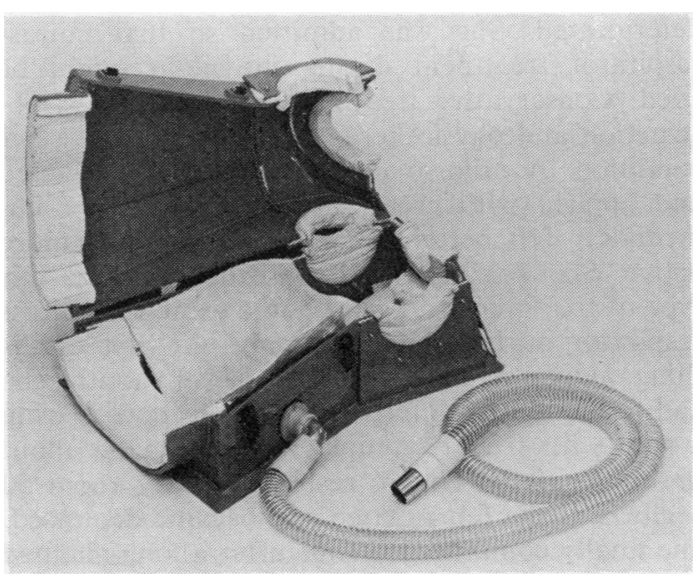

Fig. 5 Corset-cuirass which will encase the major part of the patient's trunk. the patient's thoracic cage. This shell also encases the major part of the thorax and the abdomen; it fits much better than the standard cuirass shell and the patient is able to use the apparatus without additional help. Since 1974 minor changes have been made to the shell. Since he has been using the cuirass respirator the patient has not been admitted with respiratory failure. His exercise tolerance is remarkably good; he drives his own car and can take part in many activities of daily life.

\section{CASE 1}

This patient suffered from poliomyelitis in 1957 when he was 11 years old. He received no mechanical ventilation at that time. He was discharged home with widespread pareses of the muscles of the trunk (including the intercostal muscles), shoulder girdle, and limbs. In the course

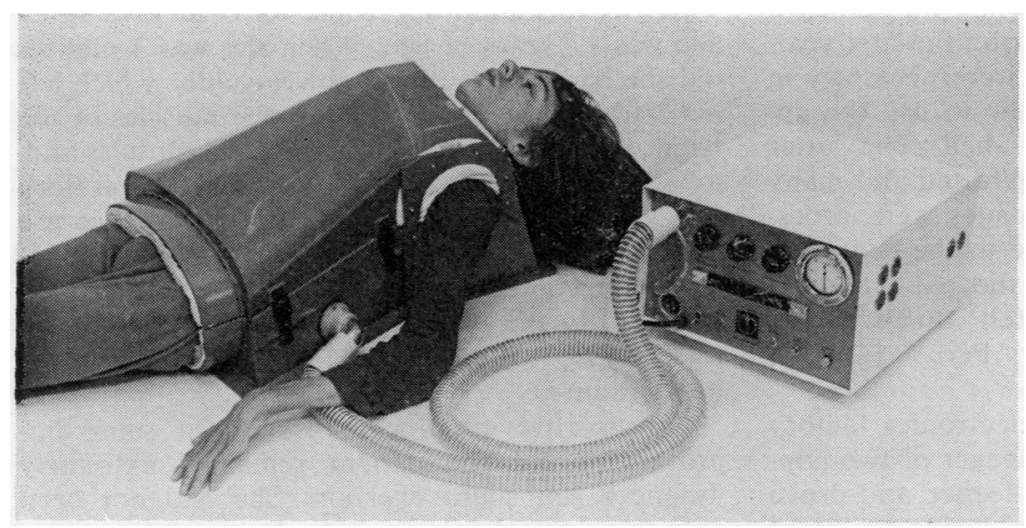

Fig. 6 Corset-cuirass in situ.

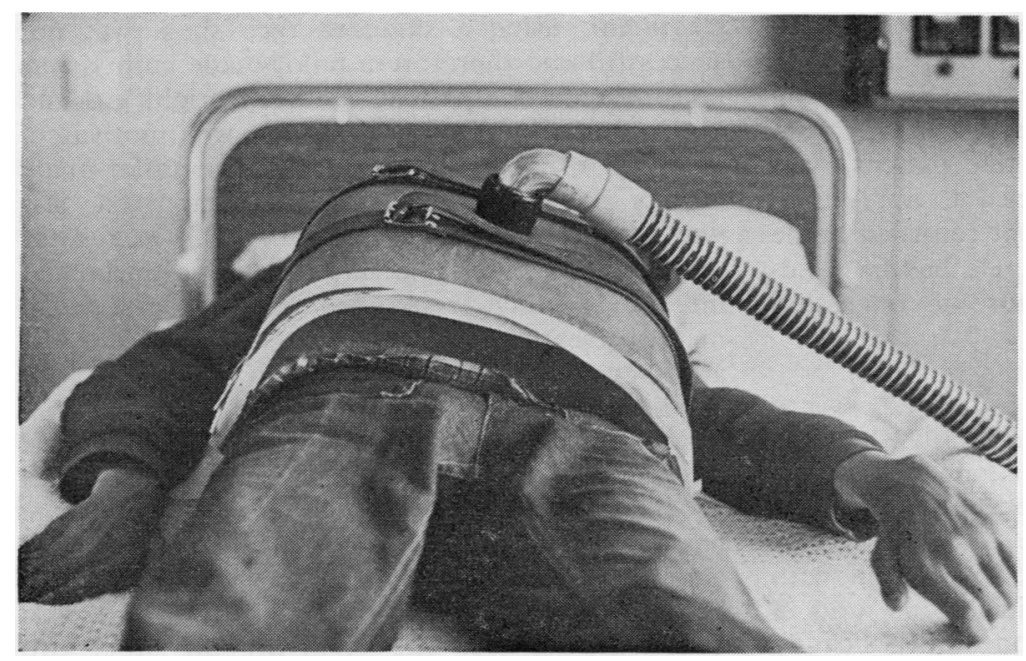

Fig. 7 Tailor-made cuirass shell in situ. 
of the next few years he developed a severe thoracic scoliosis for which a spondylodesis was performed in 1963 . He gradually started to complain of increasing dyspnoea on exertion; he also suffered from mild airways obstruction with repeated episodes of bronchial infection. In 1966 he was admitted for the first time to the RCU with cardiorespiratory failure, probably provoked by a bacterial infection. $\mathrm{He}$ was treated with conventional conservative treatment to which mechanical ventilation, first via an endotracheal tube and later via a tracheostomy, was added. He improved slowly, was weaned from the respirator and after three months was discharged home, treatment being directed towards the airways obstruction, a maintenance dose of antibiotics, and physiotherapy. Only one month later he had to be readmitted because of increasing dyspnoea. Domiciliary cuirass respirator treatment was then considered. The patient and his family were very well motivated and extensive trials, using the standard type shell, were carried out. During the first year, several admissions to the RCU were necessary in order to instruct the patient how to use the apparatus and how to apply the shell. The latter procedure was particularly complicated and many layers of cotton wool and bandages were necessary to plug all the leakages due to the deformed thoracic cage. After one year the patient could manage fairly well and he felt much better (arterial $\mathrm{Po}_{2}: 10 \mathrm{kPa}, 75$ torr; $\mathrm{PCO}_{2}: 5.7 \mathrm{kPa}$, 43 torr).

He worked part-time in an electronics factory and spent his spare time as a manager of two popgroups. He obtained a driver's licence and drove his own car. He used the cuirass during the night only. In 1974 he was again admitted to the RCU because of respiratory failure. The standard cuirass shell showed much wear and tear and, after using a new shell, the patient's condition improved. A tailor-made cuirass shell was constructed which, according to the patient, was much more efficient and more easy to apply than the standard type, and the patient returned home and resumed his daily activities. He has been admitted since then only once because of concussion following a fall.

\section{CASE 3}

This woman was first seen by us in 1972 because of progressive dyspnoea on exertion. She was at that time 41 years old and she suffered from a severe congenital scoliosis. She also had minor symptoms and signs of airways obstruction with repeated bacterial bronchial infections and a 'wheezy chest'. Her condition had steadily deteriorated. She was admitted so that cuirass respirator treatment during the night might be tried. Conservative treatment of her airways ob-C struction and physiotherapy failed to improve her condition. A tailor-made cuirass was constructed and applied with great success. The patient im $\Omega$ mediately felt better, slept better, and was mores active. She was very well motivated towards this type of treatment and went home using the cuirassrespirator during the night only. At first every $\vec{\omega}$ thing went well but after a couple of months shes had to move to a different neighbourhood. During $x$ a stay with friends complaints were made about the noise of the cuirass respirator (only about 500 decibels!); she felt isolated and became depressed $N$ She finally committed suicide after a long-planned ${ }_{-}^{-}$ holiday, on which a relative was to have accom $=$ panied her, was suddenly cancelled.

\section{CASE 4}

This girl was admitted to the RCU in 1973 whene she was 17 years of age. When she was 4 monthș old she had suffered from poliomyelitis, which left her with widespread pareses of the muscles of the trunk and shoulder girdle, intercostal muscles and to some extent, also the auxiliary respiratoryo muscles. At an early age she developed a severeD thoracic scoliosis. Several times she had broncho pneumonia and sometimes was wheezy. She was admitted to the RCU in a state of severe respira tory failure without signs or symptoms of right heart failure.

Conservative treatment resulted in some im provement but the patient remained extremely dyspnoeic on slight exertion. She had not been able to go to school regularly during the previous year because of the dyspnoea. Cuirass respirato treatment, using a standard type shell, was no successful and therefore a tailor-made cuirass was constructed. The patient is a very bright girl and both she and her parents are very well motivated She was discharged home with the tailor-made cuirass shell and felt very much improved. Sh was admitted once at the end of 1973 because of respiratory failure following a bacterial bronchia infection, which responded well to additiona medical treatment. We instructed her, her family? and her general practitioner how to deal witio colds-immediately starting with antimicrobia treatment, a short course of corticosteroids, and physiotherapy-and told her to have influenzo vaccination at least once a year. Since then sh has not been readmitted. She is doing well af home, has finished secondary school, rides $\cong$ bicycle, and is at present being trained for at administrative job. She is, as are the other tw 8 
living patients, seen regularly by us in the outpatient department.

\section{Discussion}

This retrospective, uncontrolled study on the effect of cuirass respirator treatment using tailor-made shells in severe thoracic scoliosis deals with only four patients. No definite conclusion can be drawn. The favourable results that we obtained, however, may stimulate other investigators to consider this form of treatment. We are currently engaged in more detailed investigations of the effect of this treatment.

Over the years our policy regarding treatment of scoliotic patients who present themselves with respiratory or cardiorespiratory failure has changed as far as the after-treatment is concerned. After a period in which no specific after-treatment was advised, we started to treat the patients with tracheostomy and mechanical ventilation at home; then cuirass respirator treatment using standardtype shells was used; and finally the tailor-made cuirass shell was introduced.

The natural history of thoracic scoliosis often shows a deterioration at puberty (Scadding and Zorab, 1969), especially in the paralytic forms (Lane et al., 1974). A second peak in deterioration often occurs in the fifth decade. Once respiratory failure, or cardiorespiratory failure, supervenes, the prognosis is bad: over $50 \%$ of the patients die within the next two to three years despite vigorous conservative treatment (Bergofsky et al., 1959; Towers and Zorab, 1969).

In the 17 scoliotic patients with severe respiratory insufficiency who have been treated in the RCU mortality was high in those patients who had conservative after-treatment only: of 10 patients, five died while under treatment in the RCU and another three patients died within three years of the acute episode. Seven patients received, as well as the conservative treatment, after-treatment either in the form of mechanical ventilation via a tracheostomy (3 patients, 2 survivors) or via a cuirass respirator ( 4 patients, 3 survivors, one patient committed suicide).

Lane et al. (1974) have reported the results of another technique for managing such patients using a 'dustette', a simple portable device for IPPB. The patient carries the apparatus with him during the day. In general, their patients had less severe disturbances of lung function than the patients of the present study. Mechanical ventilation via a tracheostomy is an alternative form of treatment. Although it is an effective procedure, it entails the loss of impairment of speech and a number of well-known complications, such as mucous plugging, bacterial infections, and haemorrhage. Daily chest physiotherapy is necessary and the handling of the respirator requires some technical insight and skill. Treatment with the cuirass respirator is an extremely simple procedure that can be taught to virtually any patient. The important thing is to ensure an almost airtight fitting of the cuirass shell. Formerly this was achieved by plugging all apertures with bandages and cotton wool, a rather laborious procedure. Moreover, the standard cuirass shell is liable to cause pressure sores when used upon a deformed chest. The tailor-made cuirass shell has nearly abolished these problems. After a few years some adaptations to the shell may prove to be necessary due either to wear and tear or to a change in the thoracic deformity. The patient uses the cuirass respirator only during the night; during the day he can carry out his usual activities.

We do not know how the beneficial effect of this form of treatment is achieved. It is tempting to assume that improvement of the ventilatory defect, with concurrent improvement of the ventilation/perfusion disturbance, plays an important role; part of the beneficial effect may also be due to recovery from muscular 'fatigue' during cuirass respirator treatment. Simple spirometric tracings may show some rise in tidal volume, and the patient of ten accepts a lower respiratory rate from the apparatus than his own spontaneous respiratory rate.

Detailed lung function studies to investigate this are in progress. Good motivation is essential for this treatment. Not only the patient, but also his family and relatives and society as a whole, must be willing to accept it. Instructions should be given how to deal with bacterial infections; coexistent airways obstruction should be treated vigorously, smoking must be prohibited, and influenza vaccination administered every year. Up to now we have applied domiciliary cuirass respirator treatment only in young patients with paralytic thoracic scoliosis; in these patients it works very well. We have as yet no experience with this kind of treatment in older patients suffering from scoliosis of other aetiology. We intend, however, to attempt this treatment in these patients because of the serious prognosis once respiratory failure has become apparent. Experience with cuirass treatment in older patients without scoliosis suffering from other diseases indicates that this treatment may be carried out successfully in some older scoliotic patients provided motivation is present. It seems reasonable to assume that a decreasing incidence of polio- 
myelitis will eventually result in fewer cases of severe thoracic scoliosis; moreover, it should be possible nowadays to prevent its development in most cases. In the meantime, cuirass respirator treatment, using tailor-made shells, seems worth a trial. The cost of this treatment is small compared with that of repeated hospital admissions.

We thank the medical, nursing, and technical staff of the RCU for their help in caring for these patients. We acknowledge gratefully the help of staff members of the Departments of Orthopaedics, Radiology, and Rehabilitation. We want to thank particularly Mr R. Drent, of the Technical Department of the University Hospital, for his patience, ingenuity, and technical skill in constructing the tailor-made shells.

\section{References}

Bergofsky, E. H., Turino, G. M., and Fishman, A. P. (1959). Cardiorespiratory failure in kyphoscoliosis. Medicine (Baltimore), 38, 263-317.

Bruderman, I. and Stein, M. (1961). Physiologic evaluation and treatment of kyphoscoliotic patients. Annals of Internal Medicine, 55, 94-102.

Caro, C. G. and Dubois, A. B. (1961). Pulmonary function in kyphoscoliosis. Thorax, 16, 282-290.

Dollery, C. T., Gillam, P. M. S., Hugh-Jones, P., and Zorab, P. A. (1965). Regional lung function in kyphoscoliosis. Thorax, 20, 175-181.

Fountain, F. F., Jr., Reynolds, L. B., Jr., and Tickle, S. M. (1973). Use of extra-thoracic assisted breathing in the management of chronic obstructive lung disease. American Journal of Physical Medicine, 52, 277-288.

Friedman, H. H. (1971). Diagnostic Electrocardiography and Vector Cardiography, pp. 141 and 287. McGraw-Hill Book Company, New York.

Hepper, N. G. G., Black, L. F., and Fowler, W. S. (1965). Relationships of lung volume to height and arm span in normal subjects and in patients with spinal deformity. American Review of Respiratory Diseases, 91, 356-362.

Kafer, E. R. (1975). Idiopathic scoliosis. Journal of Clinical Investigation, 55, 1153-1163.

Lane, D. J., Hazleman, B., and Nichols, P. J. R. (1974). Late onset respiratory failure in patients with previous poliomyelitis. Quarterly Journal of $\overrightarrow{\vec{c}}$ Medicine, 43, 551-568.

Lende, R. van der (1969). Epidemiology of Chronic Non-Specific Lung Disease. Thomas, Springfield, Illinois.

Lende, R. van der (1977). (1) Prevalence of chronic $\stackrel{\widehat{D}}{\circ}$ non specific lung disease (CNSLD) in Vlagtwedde and Vlaardingen. (2) Definition of CNSLD: use in ${ }^{\infty}$ epidemiology and preventive medicine. Selected $\vec{O}$ Papers of the Royal Netherlands Tuberculosis $\overrightarrow{ }$ Association, 17. (In press).

Littler, W. A., Reuben, S. R., and Lane, D. J. (1973) Lung blood flow studies in patients with scoliosis $x$ and neuromuscular weakness. Thorax, 28, 209-213. N

Mulder, J. (1956). Bacteriology of bronchitis. Proceed-i ings of the Royal Society of Medicine, 49, 773-776. N

Newson Davis, J., Goldman, M., Loh, L., and Casson, M. (1976). Diaphragm function and alveolar hypo-o ventilation. Quarterly Journal of Medicine, 45, 87-100.

Orie, N. G. M. (1961). The host factor in bronchitis. In Bronchitis, an International Symposium, edited by N. G. M. Orie and H. J. Sluiter. Royal vane Gorcum, Assen.

Prime, F. J. and Zorab, P. A. (1969). Respiratory function in scoliosis. In Scoliosis, edited by P. A.O Zorab, pp. 44-53. Heinemann, London.

Scadding, F. H. and Zorab, P. A. (1969). The lung in scoliosis. In Scoliosis, edited by P. A. Zorab, pp.气 30-43. Heinemann, London.

Tammeling, G. J. (1961). Standard values for lung $\vec{P}$ volumes and ventilatory capacity of sanatorium patients. Selected Papers of the Royal Netherlands Tuberculosis Association, 1, 65-89.

Towers, M. K. and Zorab, P. A. (1969). The heart in scoliosis. In Scoliosis, edited by P. A. Zorab, pp. 54-66. Heinemann, London.

Weber, B., Smith, J. P., Briscoe, W. A., Friedman, S. A. and King T. K. C. (1975). Pulmonary function? in asymptomatic adolescents with idiopathic scolio sis. American Review of Respiratory Diseases, 111, 389-397.

Requests for reprints to: Professor H. J. Sluiter, N Department of Pulmonary Diseases, University Hospital Groningen, 59, Oostersingel, Groningen, The ${ }^{N}$ Netherlands. 